\title{
Development and Validation of a Critical Appraisal Tool for Clinical Pharmacokinetic Research
}

\section{Alaa Soliman', Shane Pawluk ${ }^{2,3}$, Kyle Wilby ${ }^{4}$, Ousama Rachid ${ }^{1}$}

${ }^{1}$ College of Pharmacy, QU Health, Qatar University, Doha, Qatar; ${ }^{2}$ Children's \& Women's Health Centre of British Columbia, British Columbia, Canada; ${ }^{3}$ Faculty of Pharmaceutical Sciences, University of British Columbia, British Columbia, Canada; ${ }^{4}$ School of Pharmacy, University of Otago, Dunedin, New Zealand

\section{Background}

- Application of knowledge from primary literature is accompanied by enhanced ability to critically apprise primary literature and determine whether it is conducted optimally

- Quality of trials has several dimensions ${ }^{1}$.

- $20 \%$ of the published trials did not specify basic pharmacokinetic (PK) parameters that are fundamental requirements for all drug dosing ${ }^{2}$.

- Pharmacokinetic reporting guidelines were published:

To guide researchers in conducting pharmacokinetic studies ${ }^{3}$

To ensure the reporting of the required minimum basic information ${ }^{3}$

"Yes/No" checklist

The checklist was composed of 24 items

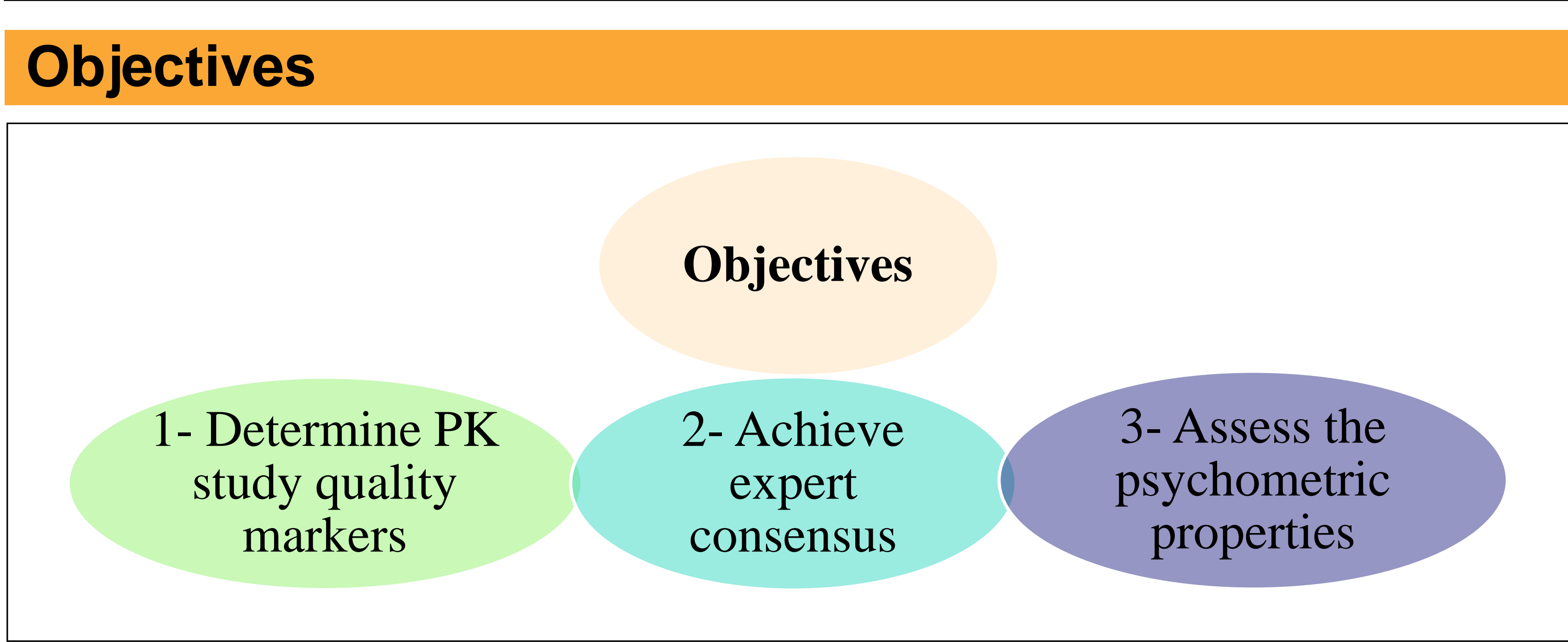

\section{Methods}

\section{Literature search}

Search of the literature published in English without years limit using Medline (via Ovid) (1946 to March 2018), EMBASE (1974 to March 2018), Cochrane databases of systematic reviews, Google and Google Scholar was conducted.

Search terms encompassed three main search categories: pharmacokinetics, reporting guidelines/appraisal tools, quality markers.

\section{Inclusion criteria:}

Primary, secondary, or tertiary levels of clinical pharmacokinetic scientific literature

- English language

Exclusion criteria:

- Cell or animal-based models

Data extraction

Data extraction form was developed

Protocol was registered with PROSPERO: CRD42018094571

\section{Modified Delphi}

Sampling method

- Purposeful sampling

Number of rounds

Determined based on the agreement, disagreements and reconsideration

\section{Consensus criteria}

\section{Inclusion criteria}

$\geq 75 \%$ of participants select 4 or 5 on the 5 -Point Likert scales.

A median score of $\geq 4$ and interquartile-range of $\leq 1$.

\section{Exclusion Criteria}

$<75 \%$ of participants select 1 or 2 on the 5 -Point Likert scales.

A median score of $\leq 2$ and interquartile-range of $>1$.

Questionnaire Development: through SurveyMonkey platform Validity and reliability testing

Content and face validity were tested

Intra-rater and inter-rater reliability were tested

\section{Limitations}

- Data collection form was not validated

- Prevalence bias lead to undetectable Kappa Values

\section{Acknowledgement}

This work was made possible by the Student Grant awards [QUST-2$\mathrm{CPH}-2018]$ from Qatar University.

\section{Results}

Figure 1. Flowchart describing study selection

Records identified through searching:

Medline, EMBASE, Cochrane databases for systematic review $\mathrm{n}=607$

Records after removing duplicates

$\mathrm{n}=600$<smiles>c1ccccc1</smiles>

Initial review of title and abstract

Records excluded $n=473$ $\mathrm{n}=600$ cited references $n=4$

Full-text articles excluded, with reasons $n=116$

Full text-articles assessed for eligibility $n=131$

$n=2$ Animal studies

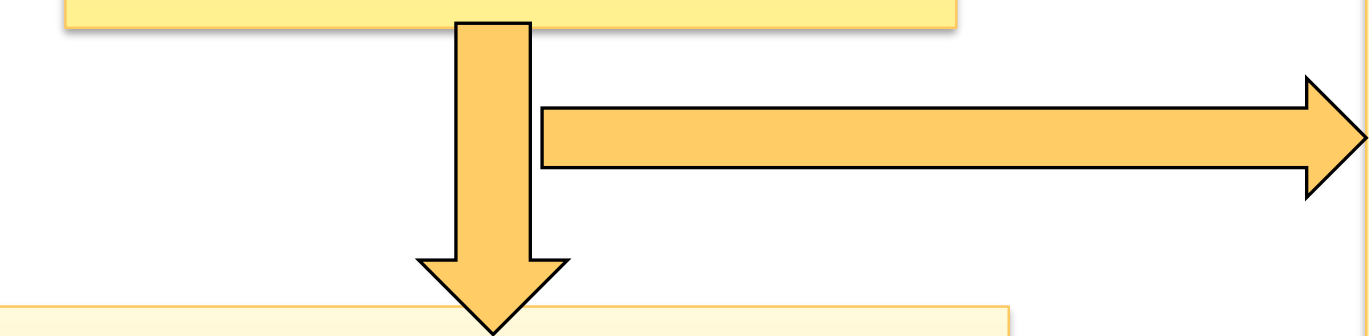

$n=7$ Full text not available

Studies included in qualitative synthesis $n=15$

to clinical PK studies

$n=3 P K$ studies from industrial perspective

$n=95$ Do not contain an item of quality

Figure 2. The Modified Delphi flow chart

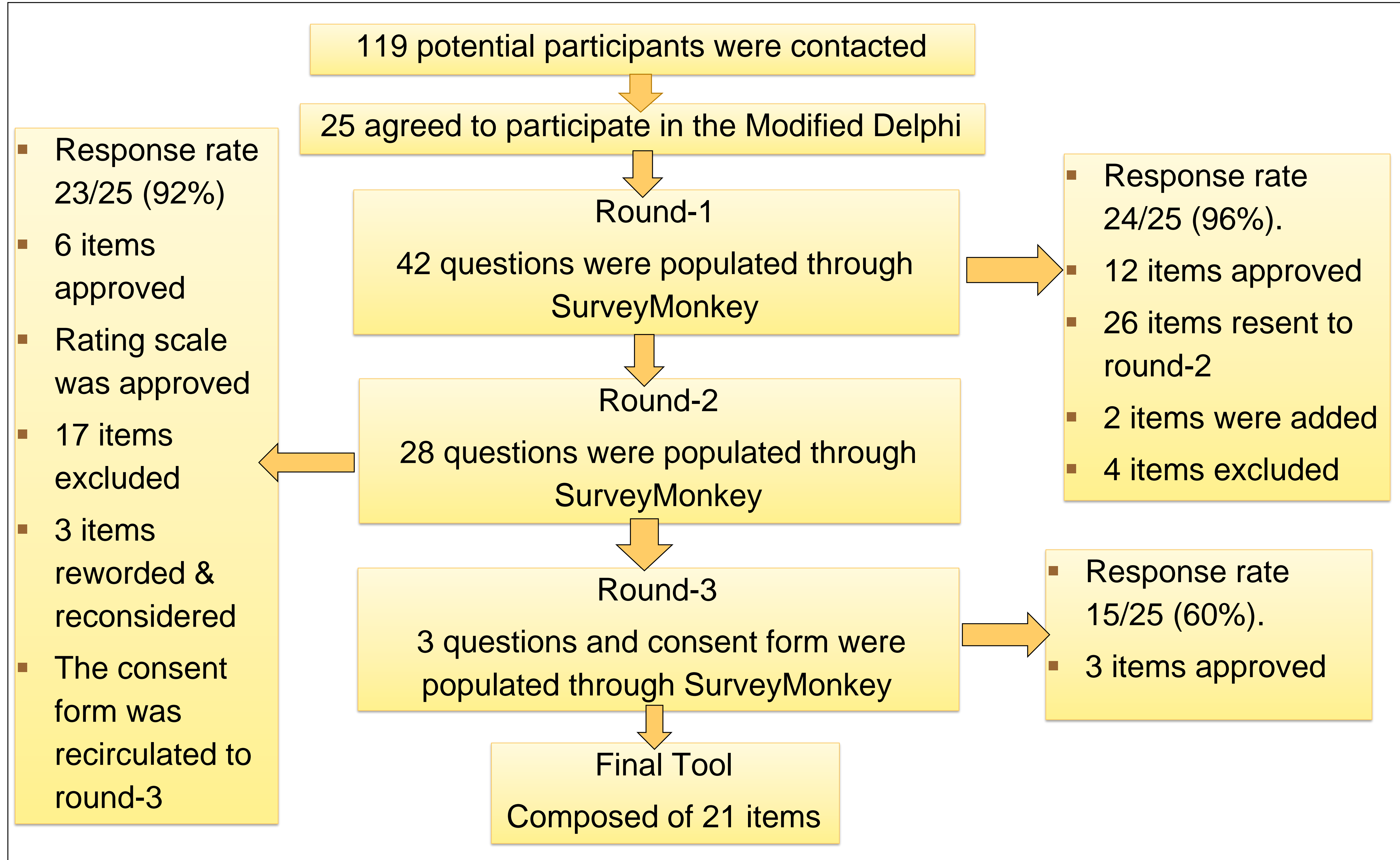

Table 1. Inter- reliability testing

$\begin{array}{ll}\text { Level of agreement } & \text { Questions (Q) } \\ \text { Less than a chance of agreement }(<0) & \text { Q3, Q10 } \\ \text { Slight agreement }(0.01-0.20) & \text { Q15 } \\ \text { Fair agreement }(0.21-0.40) & \text { Q7, Q8, Q9, Q12, Q14, Q19 } \\ \text { Moderate agreement }(0.41-0.60) & \text { Q6, Q11, Q17 } \\ \text { Substantial agreement }(0.61-0.80) & \text { Q1, Q4, Q16, Q18, Q20 } \\ \text { Almost perfect agreement }(0.81-0.99) & \text { Q13, Q21 }\end{array}$

\section{Discussion/Conclusion}

- Inventory of quality markers related to clinical pharmacokinetic studies was developed

- This critical appraisal tool will aid in enhancing the quality of the published clinical pharmacokinetic studies

\section{Future Direction}

- Further modification and psychometric testing should be done on the developed clinical pharmacokinetic critical appraisal tool to convey the dynamic evolution in the medical field.

\section{References}

1. Juni P. Altman DG, Egger M. Systematic reviews in health care: Assessing the quality of controlled clinical trials. Bmi. 2001;323(7303):42-6. 2. Li AM, Gomersall CD, Choi G, Tian Q, Joynt GM, Lipman J. A systematic review of antibiotic dosing regimens for septic patients receiving continuous renal replacement therapy: do current studies supply sufficient data? J Antimicrob Chemother. 2009;64:929-37. . Kani S, Hayes M Ling A et al Reporting guidelines for clinical pharmacokinetic studies: The ClinPK statement Clin Pharmacokinet 2015; $54 \cdot 783-95$. 